\title{
Abnormal pattern of brain glucose metabolism in Parkinson's disease: replication in three European cohorts
}

\author{
Sanne K. Meles ${ }^{1}(1) \cdot$ Remco J. Renken $^{2} \cdot$ Marco Pagani $^{3,4,5} \cdot$ L. K. Teune ${ }^{1,15} \cdot$ Dario Arnaldi $^{6,7} \cdot$ Silvia Morbelli $^{7,8}$. \\ Flavio Nobili ${ }^{6,7}$. Teus van Laar $^{1}$ • Jose A. Obeso ${ }^{9,10,11}$ • Maria C. Rodríguez-Oroz ${ }^{9,10,12,13,14} \cdot$ Klaus L. Leenders $^{5}$
}

Received: 15 July 2019 / Accepted: 3 October 2019 / Published online: 25 November 2019

(C) The Author(s) 2019

\begin{abstract}
Rationale In Parkinson's disease (PD), spatial covariance analysis of ${ }^{18} \mathrm{~F}$-FDG PET data has consistently revealed a characteristic PD-related brain pattern (PDRP). By quantifying PDRP expression on a scan-by-scan basis, this technique allows objective assessment of disease activity in individual subjects. We provide a further validation of the PDRP by applying spatial covariance analysis to PD cohorts from the Netherlands (NL), Italy (IT), and Spain (SP).

Methods The $\mathrm{PDRP}_{\mathrm{NL}}$ was previously identified (17 controls, $19 \mathrm{PD}$ ) and its expression was determined in 19 healthy controls and 20 PD patients from the Netherlands. The PDRP IT was identified in 20 controls and 20 "de-novo" PD patients from an Italian cohort. A further 24 controls and 18 "de-novo" Italian patients were used for validation. The PDRP ${ }_{\mathrm{SP}}$ was identified in 19 controls and $19 \mathrm{PD}$ patients from a Spanish cohort with late-stage PD. Thirty Spanish PD patients were used for validation. Patterns of the three centers were visually compared and then cross-validated. Furthermore, PDRP expression was determined in 8 patients with multiple system atrophy. Results A PDRP could be identified in each cohort. Each PDRP was characterized by relative hypermetabolism in the thalamus, putamen/pallidum, pons, cerebellum, and motor cortex. These changes co-varied with variable degrees of hypometabolism in posterior parietal, occipital, and frontal cortices. Frontal hypometabolism was less pronounced in "de-novo" PD subjects (Italian cohort). Occipital hypometabolism was more pronounced in late-stage PD subjects (Spanish cohort). $\mathrm{PDRP}_{\mathrm{IT}}, \mathrm{PDRP}_{\mathrm{NL}}$, and PDRP $_{\mathrm{SP}}$ were significantly expressed in PD patients compared with controls in validation cohorts from the same center $(P<$ $0.0001)$, and maintained significance on cross-validation $(P<0.005)$. PDRP expression was absent in MSA.
\end{abstract}

This article is part of the Topical Collection on Neurology

Electronic supplementary material The online version of this article (https://doi.org/10.1007/s00259-019-04570-7) contains supplementary material, which is available to authorized users.

Sanne K. Meles

s.k.meles@umcg.nl

1 Department of Neurology, University of Groningen, University Medical Center Groningen, Groningen, The Netherlands

2 Neuroimaging Center, Department of Neuroscience, University of Groningen, Groningen, The Netherlands

3 Institutes of Cognitive Sciences and Technologies, CNR, Rome, Italy

4 Department of Medical Radiation Physics and Nuclear Medicine, Karolinska University Hospital, Stockholm, Sweden

5 Department of Nuclear Medicine, University of Groningen, University Medical Center Groningen, Groningen, The Netherlands

6 Clinical Neurology, Department of Neuroscience (DINOGMI), University of Genoa, Genoa, Italy

7 IRCCS Ospedale Policlinico San Martino, Genoa, Italy
8 Nuclear Medicine, Department of Health Sciences (DISSAL), University of Genoa, Genoa, Italy

9 Neurosciences Area, CIMA, Neurology and Neurosurgery, Clínica Universidad de Navarra, Pamplona, Spain

10 Centro de Investigación Biomédica en Red sobre Enfermedades Neurodegenerativas (CIBERNED), Madrid, Spain

11 CINAC, HM Puerta del Sur, Hospitales de Madrid, and Medical School, CEU-San Pablo University, Madrid, Spain

12 Department of Neurology, Clinica Universidad de Navarra, Universidad de Navarra, Pamplona, Spain

13 BCBL. Basque Center on Cognition, Brain and Language, Donostia-San Sebastián, Spain

14 Ikerbasque, Basque Foundation for Science, Bilbao, Spain

15 Present address: Department of Neurology, Wilhelmina Ziekenhuis, Assen, Netherlands 
Conclusion The PDRP is a reproducible disease characteristic across PD populations and scanning platforms globally. Further study is needed to identify the topography of specific PD subtypes, and to identify and correct for center-specific effects.

Keywords ${ }^{18}$ F-FDG PET $\cdot$ Parkinson's disease $\cdot$ Metabolic pattern $\cdot$ Networks

\section{Introduction}

Parkinson's disease (PD) is a common neurodegenerative disorder, for which only symptomatic therapies are available. Efforts to develop neuroprotective or preventive treatments will benefit from a reliable biomarker. Ideally, such a biomarker can identify PD in its early stages, differentiate between PD and other neurodegenerative parkinsonian disorders, track disease progression, and quantify treatment effects.

In PD, abnormal accumulation of $\alpha$-synuclein in neurons impairs synaptic signaling, causing disintegration of specific neural networks [1]. Neuro-imaging with $\left[{ }^{18} \mathrm{~F}\right]$ fluorodeoxyglucose positron emission tomography $\left({ }^{18} \mathrm{~F}\right.$ FDG PET) can capture synaptic dysfunction in vivo. The radiotracer ${ }^{18} \mathrm{~F}$-FDG provides an index for the cerebral metabolic rate of glucose, which is strongly associated with neuronal activity and synaptic integrity [2].

Brain regions with altered ${ }^{18} \mathrm{~F}$-FDG uptake in PD have been identified with univariate group comparisons using Statistical Parametric Mapping (SPM) [3-7]. However, because metabolic activity is correlated in functionally interconnected brain regions, analysis of covariance is more suitable to assess whole-brain networks. Multivariate disease-related patterns can be identified with the Scaled Subprofile Model and Principal Component Analysis (SSM PCA). Subsequently, a disease-related pattern can be used to quantify the ${ }^{18} \mathrm{~F}-\mathrm{FDG}$ PET scans of new subjects [8-10]. In this procedure, an individual's scan is projected onto the pattern, resulting in a subject score. This is a single numeric value which reflects the degree of pattern expression in that individual's scan.

The PD-related pattern (PDRP) was initially identified by Eidelberg et al. with SSM PCA in 33 healthy controls and 33 PD patients from the USA [11]. This PDRP (PDRP $\left.{ }_{\text {USA }}\right)$ has served as a reference in many consecutive studies [12]. The PDRP $_{\text {USA }}$ is characterized by relatively increased metabolism of the thalamus, globus pallidus/putamen, cerebellum and pons, and by relative hypometabolism of the occipital, temporal, parietal, and frontal cortices. PDRP $\mathrm{USA}_{\text {s }}$ subject scores were significantly correlated with motor symptoms and presynaptic dopaminergic deficits in the posterior striatum [13], increased with disease progression [14], and were shown to decrease after effective treatment $[15,16]$. PDRP $\mathrm{USA}_{\text {US }}$ was significantly expressed in patients with idiopathic REM sleep behavior disorder (iRBD), a well-known prodromal disease stage of PD [17], and could discriminate between healthy controls, PD, and patients with multiple system atrophy (MSA) $[18,19]$.
Because of these properties, PDRP $_{\mathrm{USA}}$ is considered a neuro-imaging biomarker for PD [12]. It is essential that the PDRP is thoroughly validated. In collaboration with Eidelberg et al., PDRPs were identified in independent American, Indian, Chinese, and Slovenian populations [11, 15, 20, 21]. Independently from these authors, the PDRP was recently derived in an Israeli population [22]. These PDRPs were highly similar to the PDRP $_{\text {USA }}$, although minor deviations in PDRP regional topography were observed in several of these studies, which may be caused by differences in demographics or clinical characteristics of the cohorts.

We previously identified a PDRP in a retrospective cohort of PD patients scanned on dopaminergic medication [23], and subsequently in an independent cohort of prospectively included PD patients who were in the off-state $\left(\mathrm{PDRP}_{\mathrm{NL}}\right)$ [24]. We used code written in-house, and obtained similar results compared with other PDRP studies. Recently, we demonstrated significant expression of the $\mathrm{PDRP}_{\mathrm{NL}}$ in idiopathic REM sleep behavior disorder (a prodromal stage of $\mathrm{PD}$ ), $\mathrm{PD}$, and dementia with Lewy bodies [25]. However, the $\mathrm{PDRP}_{\mathrm{NL}}$ has not been validated in a larger cohort, and correlations with PDRP $_{\text {USA }}$ were not explored.

The aim of the current study was to validate the $\mathrm{PDRP}_{\mathrm{NL}}$ in several independent cohorts. We were able to test the PDRP $\mathrm{PL}_{\mathrm{NL}}$ in 19 controls and $20 \mathrm{PD}$ patients from our own clinic in the Netherlands, in 44 healthy controls and 38 "de-novo" PD patients from Italy, and 19 healthy controls and 49 late-stage PD patients from Spain. In addition, we newly identified a PDRP in Italian and Spanish datasets and performed a crossvalidation between these populations. We compared the three PDRPs to the reference pattern (PDRP $\left.{ }_{\text {USA }}\right)$.

\section{Methods}

\section{${ }^{18}$ F-FDG PET data from the Netherlands}

The $\mathrm{PDRP}_{\mathrm{NL}}$ was previously identified in ${ }^{18} \mathrm{~F}$-FDG PET scans from 17 healthy controls and 19 PD patients (NL1; Table 1) [24]. In these subjects, antiparkinsonian medication was withheld for at least $12 \mathrm{~h}$ before PET scanning.

In a previous study, we demonstrated that the $\mathrm{PDRP}_{\mathrm{NL}}$ was significantly expressed in an independent dataset of $20 \mathrm{PD}$ patients compared with 19 controls (NL2; Table 1) [25]. For the current study, we added scans of 8 patients with the parkinsonian variant of MSA (MSA-P). Patients were diagnosed with probable PD or MSA-P by a movement disorder 
Table 1 Dutch (NL) data

\begin{tabular}{|c|c|c|c|c|c|}
\hline & \multicolumn{2}{|c|}{$\mathrm{PDRP}_{\mathrm{NL}}$ derivation (NL1) data from [24] } & \multicolumn{2}{|c|}{$\mathrm{PDRP}_{\mathrm{NL}}$ validation (NL2) data from: [25] } & \multirow[t]{2}{*}{ MSA patients } \\
\hline & $\mathrm{HC}$ & $\mathrm{PD}$ & $\mathrm{HC}$ & $\mathrm{PD}$ & \\
\hline$N$ & 17 & 19 & 19 & 20 & 8 \\
\hline Age & $61.1 \pm 7.4$ & $63.7 \pm 7.5$ & $62.4 \pm 7.5$ & $67.5 \pm 8.6$ & $65 \pm 9$ \\
\hline Gender; $n$ male $\%$ & $12(71 \%)$ & $13(68 \%)$ & $9(47 \%)$ & $16(80 \%)$ & $6(75 \%)$ \\
\hline H\&Y stage $1(n)$ & & 10 & & 8 & \\
\hline H\&Y stage $2(n)$ & & 9 & & 11 & \\
\hline H\&Y stage $3(n)$ & & 0 & & 0 & \\
\hline H\&Y stage $4(n)$ & & 0 & & 1 & \\
\hline Disease duration (years) & & $4.4 \pm 3.2$ (range 1.5 to 11.5 years) & & $4.4 \pm 5.3$ & $3.8 \pm 2.3$ \\
\hline UPDRS-III (off) & & $18.4 \pm 7.4$ & & NA & NA \\
\hline MMSE (NL1) or MoCA (NL2) & $29.4 \pm 0.9$ & $28.5 \pm 1.1$ & $28.3 \pm 1.6$ & NA & NA \\
\hline Acquisition protocol & \multicolumn{5}{|c|}{$30 \mathrm{~min}$ after injection of $200 \mathrm{MBq}$ of ${ }^{18} \mathrm{~F}-\mathrm{FDG}$, scan acquisition time of 6 min. Eyes closed } \\
\hline Camera & \multicolumn{5}{|c|}{ Siemens Biograph mCT-64 } \\
\hline Reconstruction & \multicolumn{2}{|c|}{ OSEM 3D, 3i24s } & \multicolumn{3}{|c|}{$\mathrm{uHD}(\mathrm{PSF}+\mathrm{TOF}), 3 \mathrm{i} 21 \mathrm{~s}$} \\
\hline Matrix & \multicolumn{2}{|l|}{$400 \times 400$} & \multicolumn{3}{|l|}{$256 \times 256$} \\
\hline Voxel size & \multicolumn{2}{|c|}{$2.00 \times 2.03 \times 2.03$} & \multicolumn{3}{|c|}{$2.00 \times 3.18 \times 3.18$} \\
\hline Smoothing & \multicolumn{2}{|c|}{$5 \mathrm{~mm}$ FWHM; and $10 \mathrm{~mm}$ after intensity normalization } & \multicolumn{3}{|c|}{$8 \mathrm{~mm}$ FWHM } \\
\hline Medication & \multicolumn{2}{|l|}{ Off } & \multicolumn{3}{|c|}{8 off, 12 on medication } \\
\hline
\end{tabular}

Values are mean and standard deviation, unless otherwise specified

Disease duration, approximate time from first motor symptoms until scanning; $H \& Y$, Hoehn and Yahr stage; MMSE, mini-mental state examination; MoCA, Montreal Cognitive Assessment; UPDRS-III, part three of the Unified Parkinson's Disease Rating Scale (2003 version); NA, not available

specialist [26]. ${ }^{18}$ F-FDG-PET was performed in our clinic as part of routine diagnostic workup. These patients were scanned with the same camera as NL1. However, since the $\mathrm{PDRP}_{\mathrm{NL}}$ derivation study [24], reconstruction algorithms were updated (Table 1). Antiparkinsonian medication was not routinely withheld in NL2 PD patients.

\section{${ }^{18}$ F-FDG PET data from Italy}

The IT dataset consisted of ${ }^{18}$ F-FDG PET scans from 44 healthy controls and 38 consecutive outpatients with "denovo," drug-naïve PD [27] (Table 2). ${ }^{123}$ I-FP-CIT Single Photon Emission Computed Tomography (DAT SPECT) was abnormal in all Italian PD patients. Disease-related patterns are typically determined on approximately 20 patients and 20 controls. Therefore, 20 controls and 20 patients were randomly selected from the IT dataset for $\mathrm{PDRP}_{\mathrm{IT}}$ derivation. The remaining 24 controls and 18 patients were used for validation.

\section{${ }^{18}$ F-FDG PET data from Spain}

${ }^{18}$ F-FDG PET scans from 49 PD patients and 19 controls from Spain (SP) were included from a previous study (Table 3) [28]. Patients in this cohort had long disease durations and were studied in the on state (i.e., antiparkinsonian medication was continued). From this dataset, 19 PD patients were randomly selected for $\mathrm{PDRP}_{\mathrm{SP}}$ identification. The remaining 30 patients were used for validation.

\section{Identification of PDRP $_{\mathrm{NL}}, \mathrm{PDRP}_{\mathrm{IT}}$, and $\mathrm{PDRP}_{\mathrm{SP}}$}

All images were spatially normalized onto an ${ }^{18}$ F-FDG PET template in Montreal Neurological Institute brain space [29] using SPM12 software (Wellcome Department of Imaging Neuroscience, Institute of Neurology, London, UK).

Identification of the $\mathrm{PDRP}_{\mathrm{NL}}$ was described previously [24]. For identification of the PDRP $\mathrm{IT}_{\mathrm{IT}}$ and $\mathrm{PDRP}_{\mathrm{SP}}$, we applied an automated algorithm written in-house, based on the SSM PCA method of Spetsieris and Eidelberg [10], implemented in MATLAB (version 2017b; MathWorks, Natick, MA). Images were masked to remove out-of-brain voxels, log-transformed, and subject and group means were removed. Principal component analysis (PCA) was applied to the residual profiles in voxel space, and the components explaining the top $50 \%$ of the total variance were selected for further analysis. For each subject, a score was calculated on each selected principal component (PC). These scores were entered into a forward stepwise logistic regression analysis. The components that could best discriminate between controls and patients [30] were linearly combined to form the PDRP. In this linear combination, each component was weighted by the coefficient resulting 
Table 2 Italian (IT) data

Data from [27]

\begin{tabular}{|c|c|c|c|c|c|c|}
\hline & \multicolumn{2}{|c|}{ Total dataset } & \multicolumn{2}{|c|}{$\mathrm{PDRP}_{\mathrm{IT}}$ derivation } & \multicolumn{2}{|c|}{$\mathrm{PDRP}_{\mathrm{IT}}$ validation } \\
\hline & $\mathrm{HC}$ & $\mathrm{PD}$ & $\mathrm{HC}$ & PD & $\mathrm{HC}$ & PD \\
\hline$N$ & 44 & 38 & 20 & 20 & 24 & 18 \\
\hline Age & $68.8 \pm 9.7$ & $71.5 \pm 6.9$ & $68.8 \pm 9.7$ & $70.5 \pm 7.3$ & $68.8 \pm 10.0$ & $72.8 \pm 6.4$ \\
\hline Gender; $n$ male $\%$ & $32(73 \%)$ & $25(65.8 \%)$ & $14(70 \%)$ & $11(55 \%)$ & $18(75 \%)$ & $14(78 \%)$ \\
\hline H\&Y stage $1(n)$ & & 23 & & 10 & & 13 \\
\hline H\&Y stage $2(n)$ & & 15 & & 10 & & 5 \\
\hline Non-MCI $(n)$ & & 18 & & 9 & & 9 \\
\hline $\operatorname{MCI}(n)$ & & 20 & & 11 & & 9 \\
\hline PD symptom duration (months)* & & $19.3 \pm 13.6$ & & $20.5 \pm 13.3$ & & $18.4 \pm 14.4$ \\
\hline UPDRS-III (off) & & $15.2 \pm 6.9$ & & $15.5 \pm 7.3$ & & $14.9 \pm 6.4$ \\
\hline MMSE & $29.1 \pm 1.0$ & $27.7 \pm 2.3$ & $28.8 \pm 1.2$ & $27.5 \pm 2.9$ & $29.4 \pm 0.6$ & $27.9 \pm 1.1$ \\
\hline Acquisition protocol & \multicolumn{6}{|c|}{ Acquisition $45 \mathrm{~min}$ after injection of $200 \mathrm{MBq}$ of ${ }^{18} \mathrm{~F}$-FDG, scan acquisition time of $15 \mathrm{~min}$. Eyes closed. } \\
\hline Camera & \multicolumn{6}{|c|}{ Siemens Biograph 16 PET/CT } \\
\hline Reconstruction & \multicolumn{6}{|l|}{ OSEM 3D } \\
\hline Matrix & \multicolumn{6}{|l|}{$128 \times 128$} \\
\hline Voxel size & \multicolumn{6}{|c|}{$1.33 \times 1.33 \times 2.00 \mathrm{~mm}$} \\
\hline Smoothing & \multicolumn{6}{|c|}{$8 \mathrm{~mm}$ FWHM after intensity-normalization } \\
\hline Medication & \multicolumn{6}{|c|}{ Treatment naive } \\
\hline
\end{tabular}

Values are mean and standard deviation, unless otherwise specified

Disease duration, approximate time from first motor symptoms until scanning (in months); H\&Y, Hoehn and Yahr stage; MMSE, mini-mental state examination; UPDRS-III, part three of the Unified Parkinson's Disease Rating Scale (2003 version); MCI, Mild Cognitive Impairment

from the logistic regression model. All voxel weights in the PDRP were overlaid on a T1 MRI template in Montreal Neurological Institute (MNI) space for visualization. All voxels in the PDRP are used for subject score calculation.

To investigate which regions in each PDRP were stable, a bootstrap resampling was performed within each derivation set (1000 repetitions) [31]. Voxels that survived a one-sided confidence interval (CI) threshold of $90 \%$ (percentile method) after bootstrapping were overlaid on a T1 MRI template. The stable regions in the three PDRPs were visually compared.

\section{Validation of PDRP $_{\mathrm{NL}}, \mathrm{PDRP}_{\mathrm{IT}}$, and $\mathrm{PDRP}_{\mathrm{SP}}$}

For validation, subject scores for $\mathrm{PDRP}_{\mathrm{NL}}, \mathrm{PDRP}_{\mathrm{IT}}$, and $\mathrm{PDRP}_{\mathrm{SP}}$ were calculated in patients and controls from the same population. First, images were log-transformed and the subject mean and group mean (originating from the PDRP identification cohort) were removed, resulting in a residual profile for each subject. The subject score is calculated by projecting the subject residual profile on the pattern. To account for differences in data-acquisition, subject scores were always $z$-transformed to the subject scores of healthy controls that were scanned on the same camera, with identical reconstruction algorithms. If subject scores in validation PD subjects were significantly higher compared with subject scores in controls, the pattern was considered valid.

In this manner, $\mathrm{PDRP}_{\mathrm{NL}}$ subject scores were calculated in the derivation cohort (NL1) and in the validation cohort (NL2). However, data acquisition was not identical for NL1 and NL2 data. This resulted in a significant difference in PDRP $_{\mathrm{NL}}$ subject scores between the NL1 and NL2 healthy control groups (supplementary Fig 1). To correct for these differences, subject scores in NL1 were $z$-transformed to NL1 healthy controls, such that NL1 control mean was 0 with a standard deviation of 1 . Similarly, subject scores in NL2 were $z$-transformed to NL2 controls.

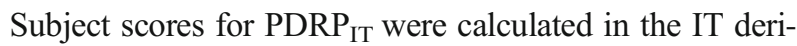
vation cohort (controls $n=20$; $\mathrm{PD} n=20$ ) and the IT validation cohort (controls $n=24$; PD $n=18$ ). Because all IT scans were acquired with identical protocols, subject scores could be $z$-transformed to the IT healthy controls from the derivation sample $(n=20)$.

Subject scores for the $\mathrm{PDRP}_{\mathrm{SP}}$ were calculated in the SP derivation cohort (controls $n=19$; PD $n=19$ ) and the SP validation cohort (PD $n=30)$. PDRP $_{\text {SP }}$ subject scores were $z$-transformed to the SP controls from the derivation sample $(n=19)$. As a second SP healthy control cohort 
Table 3 Spanish (SP) data

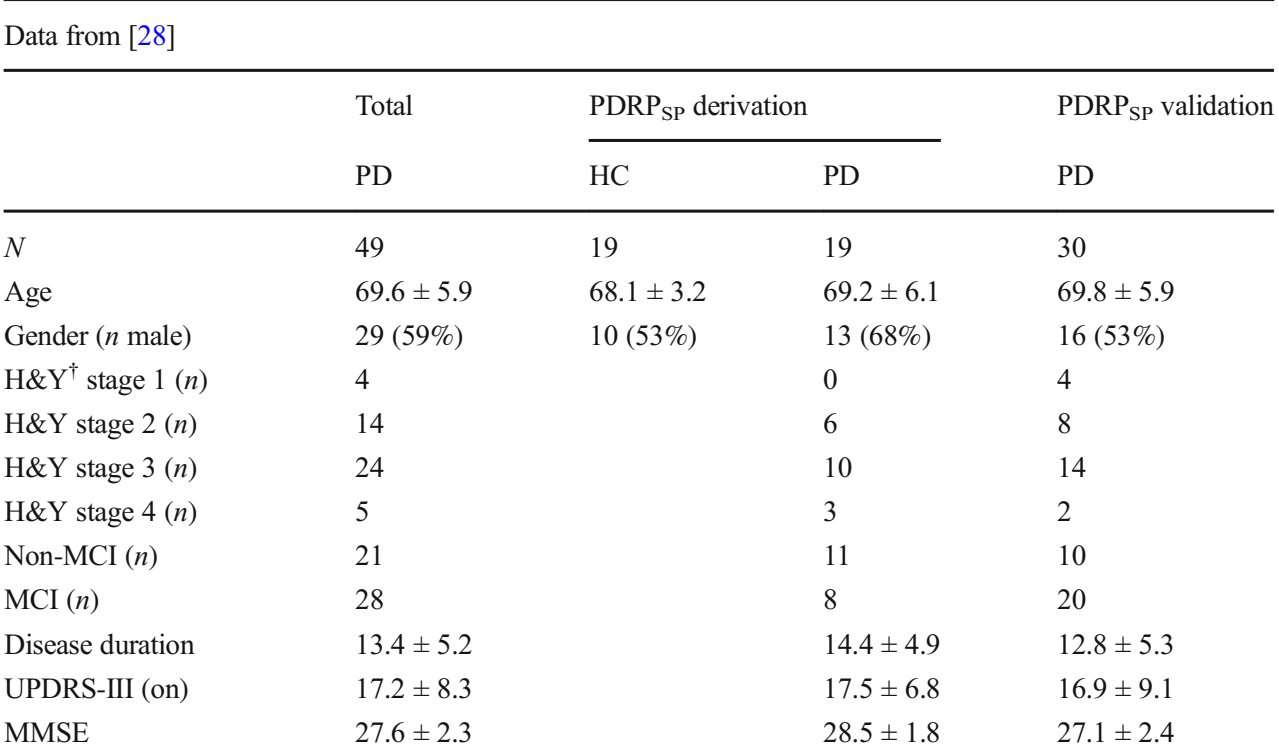

Acquisition protocol

Camera

Reconstruction

Matrix

Voxel size

Smoothing

Medication

Values are mean and standard deviation, unless otherwise specified

Disease duration, approximate time from first motor symptoms until scanning; $H \& Y$, Hoehn and Yahr stage; MMSE, mini-mental state examination; UPDRS-III, part three of the Unified Parkinson's Disease Rating Scale (2003 version); MCI, Mild Cognitive Impairment

${ }^{\dagger}$ For 2 patients in the SP dataset, H\&Y stage was not available was not available, $\mathrm{PDRP}_{\mathrm{SP}}$ subject scores in PD patients were compared with the $\mathrm{PDRP}_{\mathrm{SP}}$ subject scores in the derivation healthy controls.

\section{Cross-validation of PDRP $_{\mathrm{NL}}, \mathrm{PDRP}_{\mathrm{IT}}$, and PDRP}

Subsequently, PDRP $\mathrm{P}_{\mathrm{NL}}$ subject scores were determined in the IT and SP datasets, PDRP ${ }_{\text {IT }}$ subject scores were determined in the NL and SP datasets, and PDRP $\mathrm{SP}_{\mathrm{SP}}$ subject scores were determined in the NL and IT datasets. In addition, subject scores for the PDRP $\mathrm{PSA}_{\text {USA }}$ were calculated in each dataset in the same manner. Each subject score was then transformed into a $z$-score with respect to controls from the same camera, such that control mean was 0 with a standard deviation of 1 . To determine the performance of each pattern in discriminating between controls and patients, a receiver operating curve was plotted (for each pattern in each dataset) and the area under the curve (AUC) was obtained.

The similarity of the three PDRPs to each other and to the PDRP $\mathrm{USA}_{\text {A }}$ was tested in two ways. First, in each dataset, the $z$-scores for each PDRP were correlated with Pearson's $r$ correlation coefficient. Second, the voxelwise topographies of the different patterns were compared by using volume-of-interest (VOI) correlations over the whole brain. A set of 30 standardized VOIs were selected from a previous study [21,32], reflecting key nodes of the reference PDRP. Within each VOI, region weights were 
calculated for each pattern. Subsequently, region weights between any two of the patterns were correlated using Pearson's $r$ correlation coefficient.

\section{PDRP expression in MSA-p subjects}

Subject scores for each PDRP were calculated in 8 MSA-p patients. Subject scores for each PDRP were $z$-transformed to corresponding subject scores in NL2 controls.

\section{Principal component 1}

PDRP $_{\text {USA }}$ [11], as well as the PDRP determined in Chinese [20] and Slovenian [21] populations, consisted of $\mathrm{PC} 1$ in isolation. Combinations of components were not considered. There are several methods to decide which components are disease-related and should be included in the final diseaserelated pattern [10]. In the current study, this decision was based on a forward stepwise logistic regression model, using the Akaike information criterion (AIC) as model selection criterion [30], in order to combine the least possible number of components to obtain the optimum discrimination between controls and patients. It is possible that the optimal model selects one component. If the PDRPs identified in the current study were not based on PC1 in isolation, we repeated all analyses using $\mathrm{PC} 1$ alone for each cohort. In that case, we additionally identified $\mathrm{PDRP}_{\mathrm{NL}}-\mathrm{PC} 1, \mathrm{PDP}_{\mathrm{IT}}-\mathrm{PC} 1$, and $\mathrm{PDRP}_{\mathrm{SP}} \mathrm{PC} 1$, and repeated the cross-validation.

\section{Statistical procedures}

Between-group differences in PDRP $z$-scores were assessed using a Student's $t$ test. Correlations between PDRP and age, disease duration, and UPDRS were examined with Pearson's $r$ correlation coefficient. Analyses were performed using SPSS software version 20 (SPSS Inc., Chicago, IL) and considered significant for $P<0.05$ (uncorrected).

\section{Results}

\section{PDRP $_{N L}$}

The first six principal components explained $50 \%$ of the total variance. The $\mathrm{PDRP}_{\mathrm{NL}}$ was formed by a weighted linear combination of principal components 1 and 2 (variance explained $17 \%$ and $10 \%$, respectively; Figs. $1 \mathrm{a}$ and $2 \mathrm{a}) . \mathrm{PDRP}_{\mathrm{NL}} z-$ scores were significantly different between healthy controls and PD patients in both derivation (NL1) and validation (NL2) cohorts $(P<0.0001 ;$ Fig. 3a).
PDRP $_{\text {IT }}$

The first six principal components explained $51 \%$ of the total variance. A weighted linear combination of principal components 1 and 2 (variance explained 19\% and 8\% respectively) could best discriminate between controls and patients in the logistic regression model, and was termed the PDRP $\mathrm{IT}_{\mathrm{IT}}$ (Figs. $1 \mathrm{~b}$ and $2 \mathrm{~b}$ ). PDRP $\mathrm{IT}_{\mathrm{IT}}$ subject scores were significantly different between healthy controls $(n=24)$ and patients $(n=18)$ in the validation cohort $(P<0.0001$; Fig. 3b).

\section{PDRP $_{S P}$}

The first five principal components explained $51 \%$ of the total variance. The PDRP $\mathrm{SP}_{\mathrm{SP}}$ was formed by a weighted linear combination of principal components 1,2 , and 3 (variance explained $17 \%, 14 \%$, and 5\%, respectively; Figs. 1c and 2c). PDRP $_{\mathrm{SP}}$ was significantly expressed in PD patients from the validation set $(P<0.0001$, Fig. $3 c)$.

\section{Cross-validation}

Each of the PDRPs (including the PDRP $\mathrm{USA}_{\mathrm{UA}}$ ) was significantly expressed in $\mathrm{PD}$ patients compared with controls, in each of the datasets (Figs. 4a-c and 5). Corresponding ROC-AUCs are reported in Table 4.

Correlations to UPDRS and disease duration were inconsistent (Table 5). Within each dataset, $z$-scores of any two PDRPs were significantly correlated. Subject scores on all three patterns were also significantly correlated to subject scores on $\mathrm{PDRP}_{\mathrm{USA}}$ (Table 5). Especially, the PDRP $\mathrm{NL}_{\mathrm{NL}}$

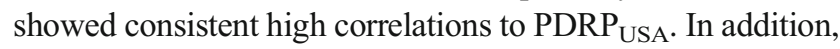
a comparison between spatial topographies of the original PDRP $_{\text {USA }}$ versus the PDRP $P_{\text {IT }}$, PDRP $_{\mathrm{NL}}$, and PDRP $\mathrm{SP}_{\mathrm{SP}}$ showed significant correlations in region weights (Table 6).

\section{PDRP subject scores in MSA-p patients}

Subject scores for each PDRP were calculated in MSA patients. Subject $z$-scores on each PDRP were not significantly different between controls and MSA patients (Fig. 6).

\section{Principal component 1}

As stated above, PDRP $_{\mathrm{NL}}$ and PDRP $\mathrm{IT}_{\mathrm{T}}$ were identified as linear combinations of multiple PCs. All analyses were repeated for $\mathrm{PDRP}_{\mathrm{NL}}-\mathrm{PC} 1, \mathrm{PDP}_{\mathrm{IT}}-\mathrm{PC} 1$, and $\mathrm{PDRP}_{\mathrm{SP}^{-}}$ PC1. The PDRPs that were based on combinations of PCs yielded higher diagnostic accuracy (Table 4) compared with patterns based on PC1 alone (Table 7). However, subject scores on $\mathrm{PDP}_{\mathrm{IT}} \mathrm{PC} 1, \mathrm{PDRP}_{\mathrm{NL}}-\mathrm{PC} 1$, and $\mathrm{PDRP}_{\mathrm{SP}} \mathrm{PC} 1$ did show much higher correlations to subject scores on $\mathrm{PDRP}_{\mathrm{USA}}$ (all $r>0.98, P<0.0001$ ). 

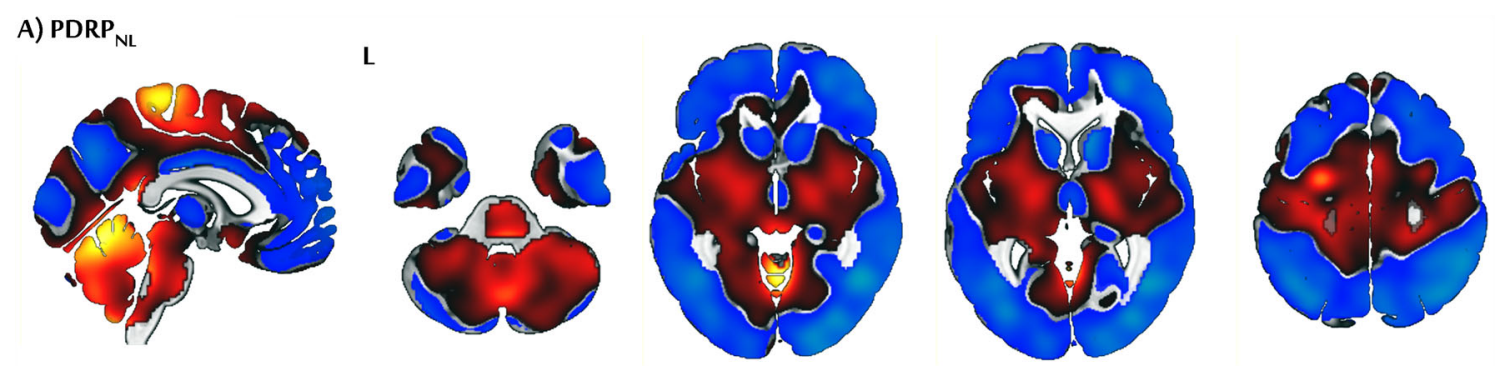

A) PDRP
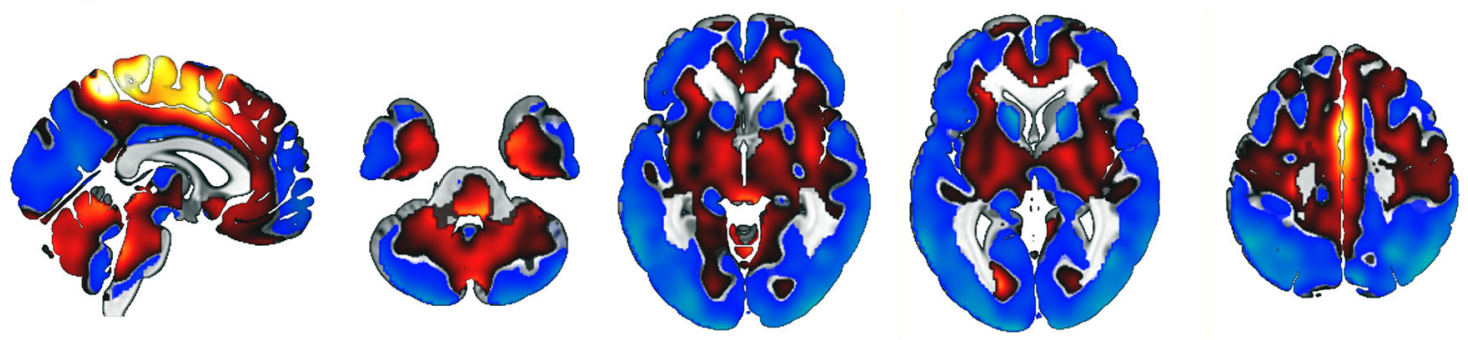

A) PDRP
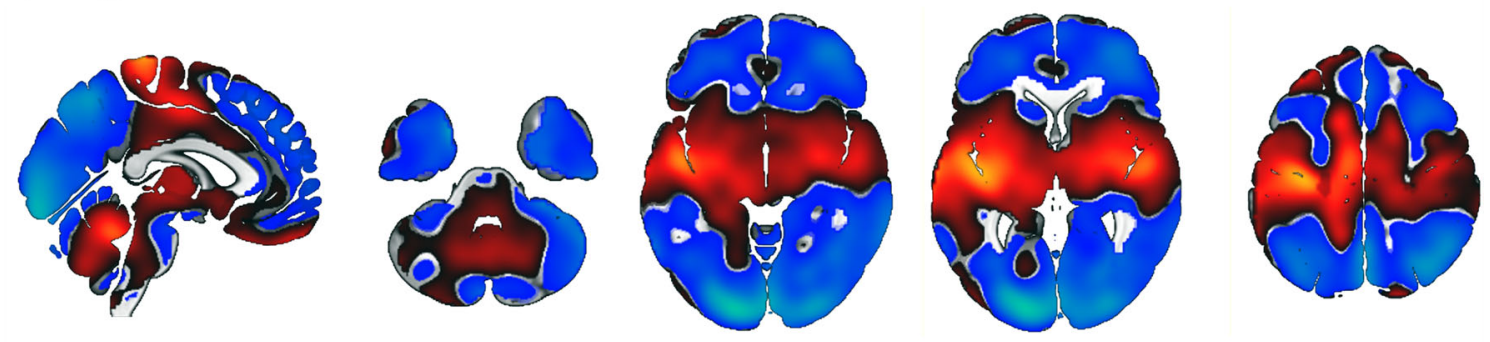

$$
x=-2
$$

$$
Z=-36
$$

$Z=-4$

$Z=2$

$Z=45$

Fig. 1 Display of $\operatorname{PDRP}_{\mathrm{NL}}(\mathbf{a}), \mathrm{PDRP}_{\mathrm{IT}}(\mathbf{b})$, and $\mathrm{PDRP}_{\mathrm{SP}}(\mathbf{c})$. All voxel values of each PDRP are overlaid on a T1 MRI template. Red indicates positive voxel weights (relative hypermetabolism) and blue indicates negative voxel weights (relative hypometabolism). $\mathrm{L}=$ left. Coordinates in the axial $(\mathrm{Z})$ and sagittal $(\mathrm{X})$ planes are in Montreal Neurological Institute (MNI) standard space.

\section{Discussion}

In this study, we cross-validated the previously published $\mathrm{PDRP}_{\mathrm{NL}}$ [24], and additionally identified a PDRP in an Italian $\left(\mathrm{PDRP}_{\mathrm{IT}}\right)$ and Spanish $\left(\mathrm{PDRP}_{\mathrm{SP}}\right)$ sample. The three patterns were akin to PDRP $\mathrm{USA}_{\text {, }}$, and also to the PDRP described in other populations [20,21]. Topographical similarity

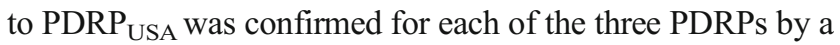
significant correlation of region weights, and a significant correlation in subject scores. Furthermore, $\mathrm{PDRP}_{\mathrm{NL}}, \mathrm{PDRP}_{\mathrm{IT}}$, and PDRP $_{\mathrm{SP}}$ were significantly expressed in PD patients compared with controls in both identification and validation cohorts, but were not significantly expressed in MSA-p patients.

The typical PDRP topography is characterized by relative hypermetabolism in the thalamus, putamen/pallidum, pons, cerebellum, and motor cortex. These changes co-vary with relatively decreased metabolism in the prefrontal, parietal, temporal, and occipital cortices [11, 15, 20, 21, 23, 24]. This topography is thought to reflect changes in corticostriatopallido-thalamocortical (CSPTC) loops and related pathways in PD [33, 34]. In these circuits, a dopaminergic deficit leads to abnormal basal ganglia output, mediated by hyperactivity of the subthalamic nucleus (STN) and its efferent projections. Several studies support a direct relationship between altered STN output and the PDRP topography [16, 35-38].

The high degree of similarity in PDRP topography across samples is striking considering differences in demographics, clinical characteristics, scanning methods, and reconstruction algorithms. Especially the $\mathrm{PDRP}_{\mathrm{NL}}$ was highly similar to the reference pattern $\left(\mathrm{PDRP}_{\mathrm{USA}}\right)$. These two patterns showed the highest subject score correlation and region weight correlation. Furthermore, the $\mathrm{PDRP}_{\mathrm{NL}}$ achieved the highest AUC in the other cohorts. Like PDRP ${ }_{\mathrm{USA}}, \mathrm{PDRP}_{\mathrm{NL}}$ was derived in a cohort of off-state patients with a wide range of disease durations (duration $4.4 \pm 3.2$ years; range $1.5-11.5$ years) and severities.

Deviations from the typical PDRP topography are worth exploring further in relation to clinical characteristics. The $\mathrm{PDRP}_{\mathrm{IT}}$ is unique in that it is, to our knowledge, the first time the PDRP has been derived in "de-novo," treatment-naïve PD patients. It is likely that these very early-stage patients have a 

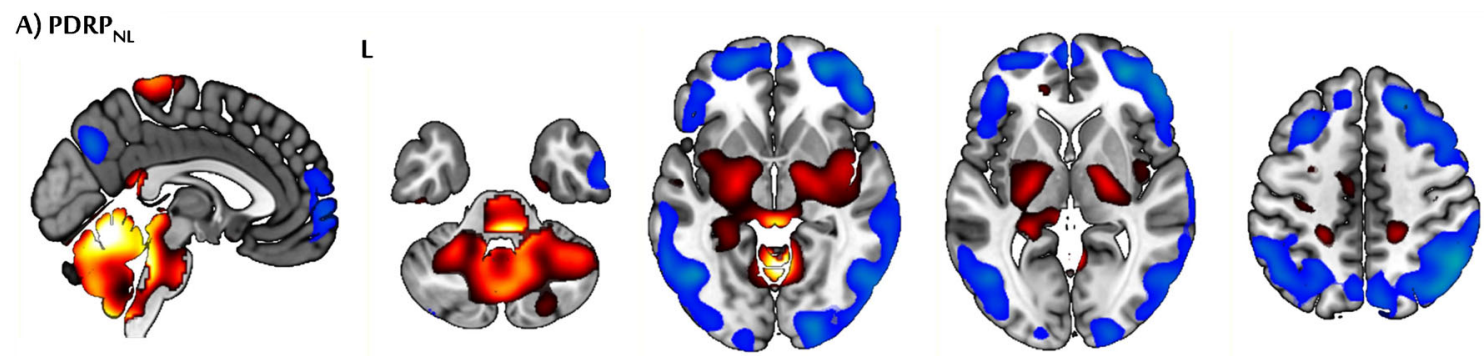

A) PDRP
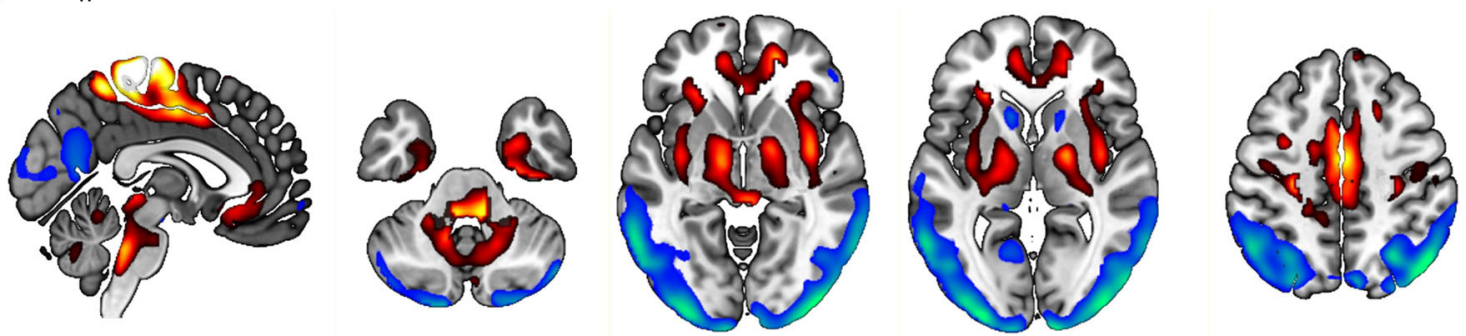

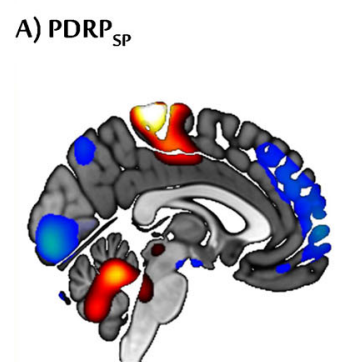

$x=-2$

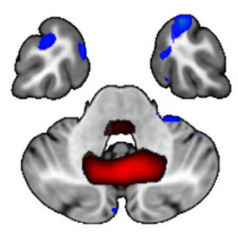

$Z=-36$

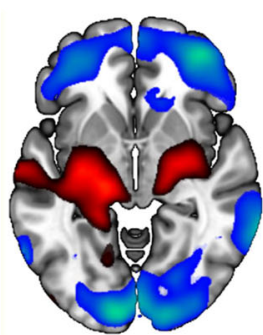

$Z=-4$

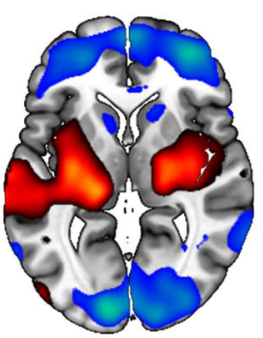

$Z=2$

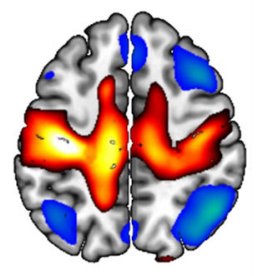

$Z=45$

Fig. 2 Display of stable voxels of each PDRP, determined after bootstrap resampling (90\% confidence interval not straddling zero). Overlay on a T1 MRI template. Positive voxel weights are color-coded red (relative hypermetabolism), and negative voxel weights are color-coded blue

less severe nigrostriatal dopaminergic deficit compared with the more advanced PD patients in $\mathrm{PDRP}_{\mathrm{USA}}, \mathrm{PDRP}_{\mathrm{NL}}$, and $\mathrm{PDRP}_{\mathrm{SP}}$ derivation cohorts. This may be reflected by less severe involvement of the frontal cortex in PDRP ${ }_{\mathrm{IT}}$, as nigrostriatal denervation is known to be positively correlated with hypometabolism in the frontal cortex $[13,39]$.

The PDRP ${ }_{\mathrm{SP}}$ was derived in PD patients who were scanned while being on dopaminergic medication. Levodopa is known to decrease metabolism in the cerebellar vermis, putamen/ pallidum, and sensorimotor cortex. Levodopa therapy can reduce PDRP expression, but does not completely correct the underlying network abnormalities [16]. It can be assumed that the effect of dopaminergic therapy on PDRP expression is modest in comparison with the effect of disease progression [40]. Indeed, the typical PDRP topography could still be identified in these $o n$-state patients. However, the PDRP ${ }_{\mathrm{SP}}$ did not correlate as well to the other patterns, both in terms of subject scores and region weights. It is not clear whether this is related to the advanced disease stage or the effect of treatment. The $\mathrm{PDRP}_{\mathrm{SP}}$ was characterized by more stable involvement of the occipital cortex, possibly related to the presence of mild (relative hypometabolism). L, left. Coordinates in the axial $(\mathrm{Z})$ and sagittal (X) planes are in Montreal Neurological Institute (MNI) standard space.

cognitive impairment and visual hallucinations, which often occur in advanced PD [41].

Following from the above, it can be concluded that the typical PDRP topography is highly reproducible. Similar topographies have also been identified in studies comparing ${ }^{18} \mathrm{~F}$-FDGPET scans of healthy controls and PD patients with SPM [3-7]. Such analyses can be supportive in the visual assessment of an ${ }^{18} \mathrm{~F}$-FDG-PET scan in clinical practice. Several studies have evaluated the diagnostic value of observer-dependent visual reads supported by SPM-based comparisons to healthy controls [3, 4, 42-44]. A recent meta-analysis (PD versus "atypical" parkinsonism) estimated a pooled sensitivity of $91.4 \%$ and specificity of $94.7 \%$ for this semi-quantitative approach [45].

The merit of SSM PCA over mass-univariate approaches lies in its ability to objectively quantify ${ }^{18}$ F-FDG PET scans of patients using the pre-defined patterns. Pattern expression scores were shown useful in differential diagnosis, tracking disease progression, and estimating treatment effects [46]. Although in the current study PDRP $z$-scores were significantly higher in PD patients compared with healthy controls, there was a considerable overlap in PDRP $z$-scores between patients 


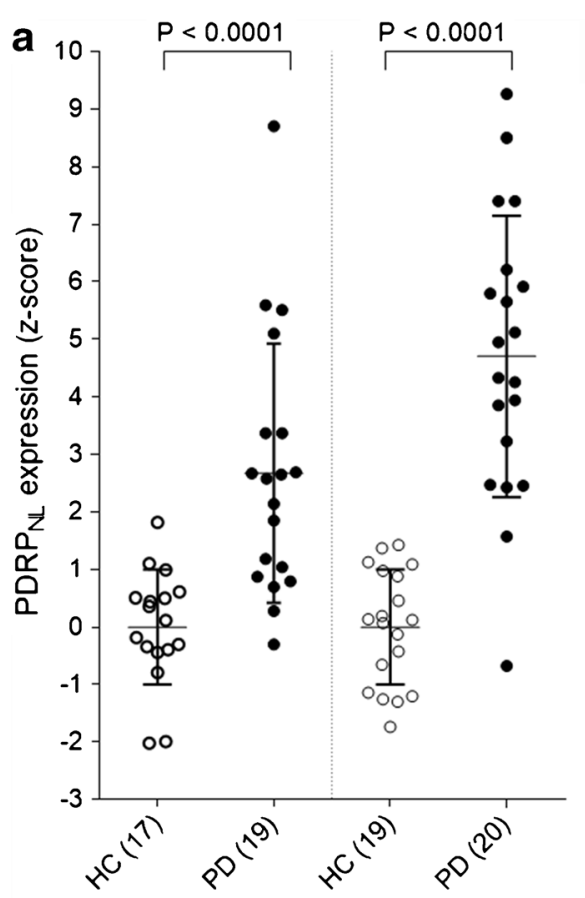

NL1 derivation

NL2 validation

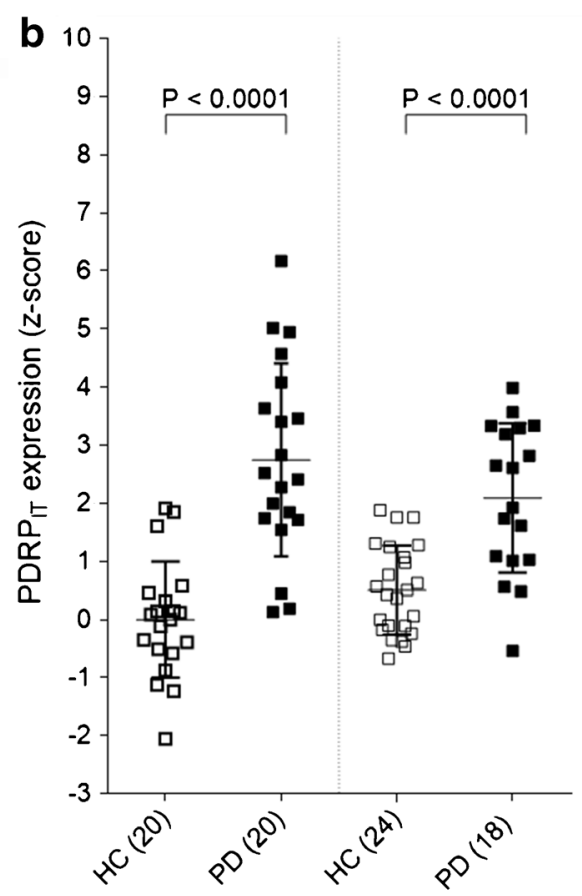

IT derivation

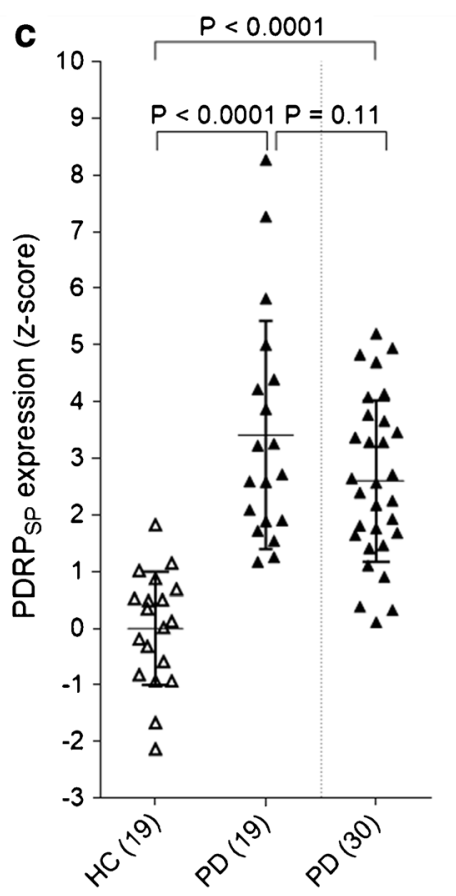

SP derivation SP validation
Fig. 3 Subject scores for each PDRP in their respective derivation and validation cohorts. a PDRP $\mathrm{NL}_{\mathrm{N}}$ was identified in $17 \mathrm{HC}$ and $19 \mathrm{PD}$ (NL1) and validated in $19 \mathrm{HC}$ and $20 \mathrm{PD}$ (NL2). Because reconstruction parameters were different for cohort NL1 and NL2, PDRP subject scores were $z$-transformed to corresponding healthy controls. b PDRP IT was identified in $20 \mathrm{HC}$ and $20 \mathrm{PD}$, and validated in $24 \mathrm{HC}$ and $18 \mathrm{PD}$.
All subject scores were $z$-transformed to the $20 \mathrm{HC}$ from the derivation sample. $\mathbf{c} \mathrm{PDRP}_{\mathrm{SP}}$ was identified in $19 \mathrm{HC}$ and $19 \mathrm{PD}$, and validated in 30 PD. Additional $\mathrm{HC}$ for validation were not available. All subject scores were $z$-transformed to the 19 HC from the derivation sample. Subject $z$ scores are compared between groups with a Student's $t$ test. Bars indicate mean and standard deviation

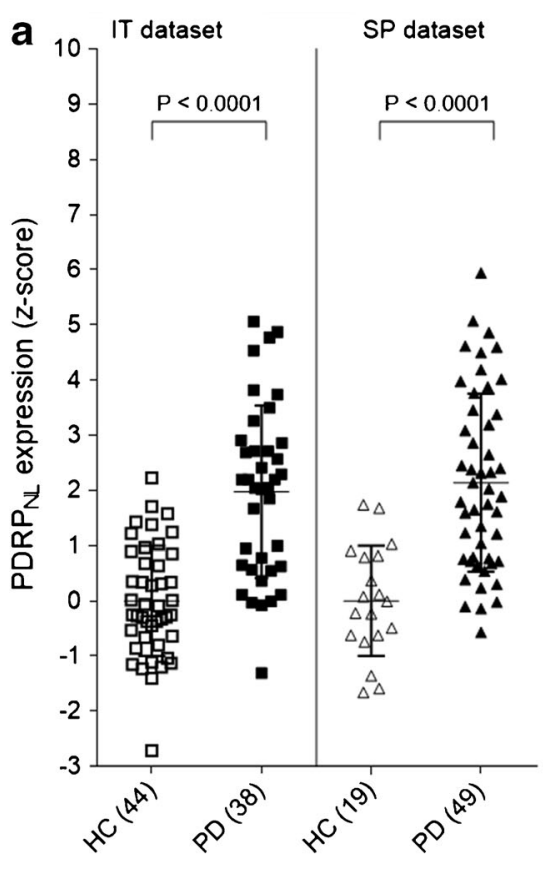

Fig. 4 Subject scores for each PDRP in the other cohorts (crossvalidation). a PDRP $\mathrm{PL}_{\mathrm{NL}}$ subject scores are plotted for the Italian (IT) and

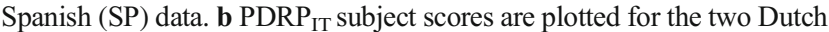
samples (NL1 and NL2) and in SP data. PDRP $_{\mathrm{SP}}$ subject scores are
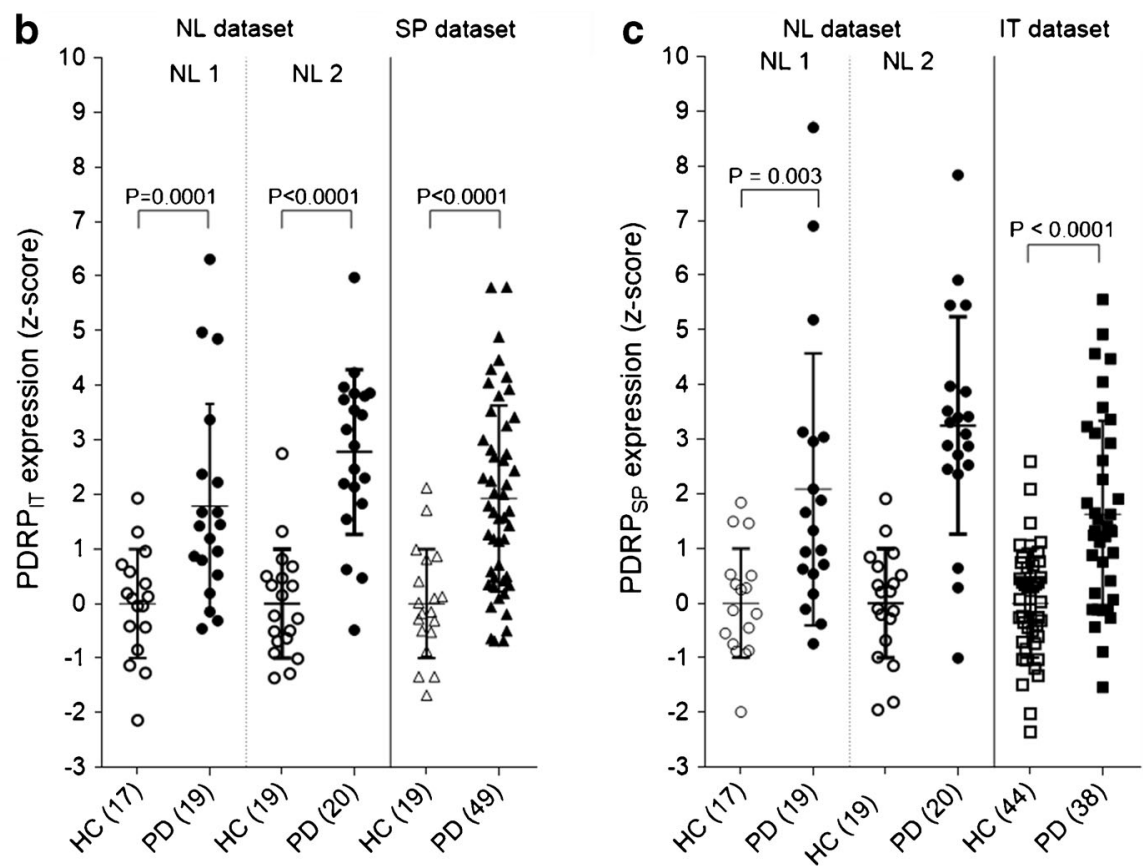

plotted for NL1, NL2, and IT data. Subject scores are $z$-transformed to healthy control values from the same camera, and compared between groups with a Student's $t$ test. Bars indicate mean and standard deviation 
Fig. 5 Subject $z$-scores for the reference pattern PDRP $_{\mathrm{USA}}$ [11] in each of the datasets. Subject scores are $z$-transformed to healthy control values from the same camera, and compared between groups with a Student's $t$ test. Bars indicate mean and standard deviation

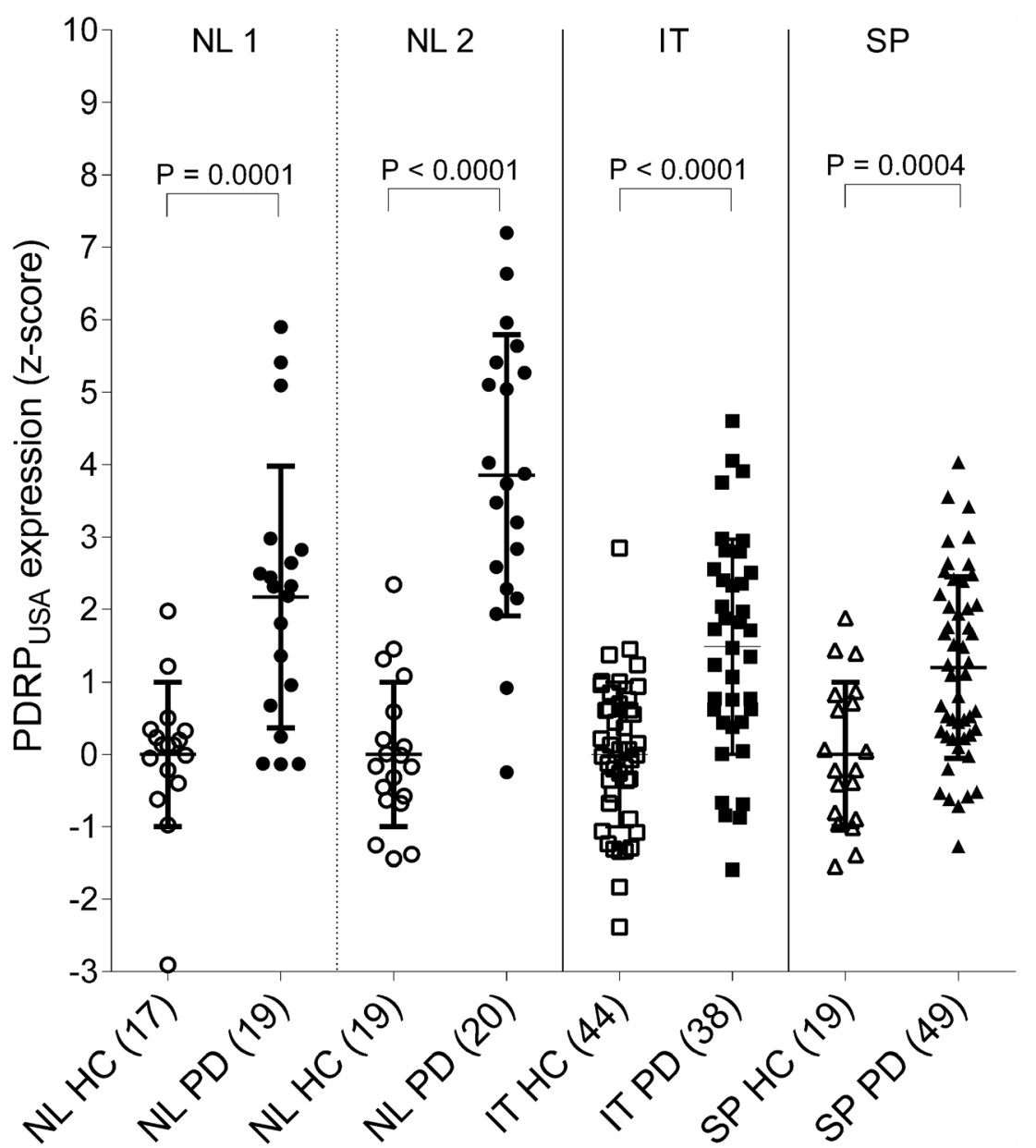

and controls in almost every cohort. This overlap is not unique to the current data, and is also apparent in other studies [12].

Some healthy controls appear to express the PDRP. Since we found significant correlations between PDRP $z$-scores and age in healthy controls, it could be suggested that ageing and PD share certain metabolic features. Metabolic decreases have been reported in the parietal cortex in normal aging $[47,48]$. This may cause some overlap with the PDRP. However, the correlation with age in our study was not consistent across all datasets and patterns

Table 4 Cross-validation of patterns

\begin{tabular}{lllll}
\hline & NL dataset 1 & NL dataset 2 & IT dataset & SP dataset \\
\hline N HC/PD $17 / 19$ & $19 / 20$ & $44 / 38$ & $19 / 49$ \\
PDRP $_{\mathrm{NL}}$ AUC & & 0.96 & 0.86 & 0.87 \\
PDRP $_{\mathrm{IT}}$ AUC & 0.81 & 0.93 & $0.83^{\dagger}$ & 0.83 \\
PDRP $_{\mathrm{SP}}$ AUC & 0.82 & 0.92 & 0.80 & \\
PDRP $_{\text {USA }}$ AUC & 0.85 & 0.95 & 0.79 & 0.76 \\
\hline
\end{tabular}

Subject scores for each PDRP were obtained in each dataset and subsequently $z$-transformed (see Figs. 3 and 4). With these scores, a receiver operating curve was plotted (for each pattern in each dataset) and the area under the curve (AUC) was obtained

${ }^{\dagger}$ Obtained from the IT validation cohort $(\mathrm{HC}=24 ; \mathrm{PD}=18)$
(Table 5). Furthermore, expression of an age-related spatial covariance pattern was shown to be independent from PDRP expression [49, 50]. Alternatively, a high PDRP z-score in a healthy subject could signal a prodromal stage of neurodegeneration. For instance, subjects with idiopathic REM sleep behavior disorder (a prodromal stage of PD) were shown to express the PDRP years before onset of clinical parkinsonism [17, 25].

Low PDRP $z$-scores in PD patients could indicate inaccurate clinical diagnosis. A recent meta-analysis of clinicopathologic studies suggests that the clinical diagnosis of PD by an expert, after an adequate follow-up, has a sensitivity of $81.3 \%$ and a specificity of $83.5 \%$ [51]. Thus, even under ideal circumstances, the diagnosis is inaccurate in a number of patients.

Overlap in pattern expression scores is not only apparent between controls and PD patients, but also between patients with different parkinsonian disorders. For instance, the PDRP may also be expressed in patients with progressive supranuclear palsy (PSP) [52]. This means that the expression score for a single disease-related pattern is inadequate to differentiate between multiple disorders. However, this does not hamper application in differential diagnosis. Previous studies have shown that an algorithm combining multiple disease- 
Table 5 Correlations between PDRP subject scores and clinical data

\begin{tabular}{|c|c|c|c|c|c|c|c|c|}
\hline \multicolumn{9}{|l|}{ NL data } \\
\hline & Age (HC) & Age (PD) & Disease duration & UPDRS (off) & $\mathrm{PDRP}_{\mathrm{NL}}$ & $\mathrm{PDRP}_{\mathrm{IT}}$ & $\mathrm{PDRP}_{\mathrm{SP}}$ & PDRP $_{\text {USA }}$ \\
\hline \multicolumn{9}{|l|}{ NL1 } \\
\hline $\mathrm{PDRP}_{\mathrm{IT}}$ & -0.02 & 0.24 & $0.50 *$ & 0.38 & & & $0.84 * * *$ & $0.79 * * *$ \\
\hline $\mathrm{PDRP}_{\mathrm{SP}}$ & 0.16 & 0.20 & $0.50^{*}$ & 0.42 & & $0.84 * * *$ & & $0.71 * * *$ \\
\hline PDRP $_{\text {USA }}$ & $0.64 * *$ & $0.50^{*}$ & $0.60 * *$ & $0.49^{*}$ & & $0.79 * * *$ & $0.71 * * *$ & \\
\hline \multicolumn{9}{|l|}{ NL2 } \\
\hline $\mathrm{PDRP}_{\mathrm{NL}}$ & 0.20 & $0.590 * *$ & 0.087 & NA & & $0.89 * * *$ & $0.76 * * *$ & $0.93 * * *$ \\
\hline $\mathrm{PDRP}_{\mathrm{IT}}$ & 0.07 & 0.387 & 0.229 & NA & $0.89 * * *$ & & $0.87 * * *$ & $0.75 * * *$ \\
\hline $\mathrm{PDRP}_{\mathrm{SP}}$ & 0.13 & $0.459^{*}$ & 0.102 & NA & $0.76^{* * * *}$ & $0.87 * * *$ & & $0.72 * * *$ \\
\hline $\mathrm{PDRP}_{\text {USA }}$ & $0.46^{*}$ & $0.698 * *$ & 0.070 & NA & $0.93 * * *$ & $0.75^{* * *}$ & $0.72 * * *$ & \\
\hline \multicolumn{9}{|l|}{ IT data } \\
\hline $\mathrm{PDRP}_{\mathrm{NL}}$ & 0.30 & $0.48 * *$ & 0.04 & $0.35 *$ & & $0.87 * * * \dagger$ & $0.73 * * *$ & $0.92 * * *$ \\
\hline $\operatorname{PDRP}_{\mathrm{IT}}$ & $0.34^{\dagger}$ & $0.23^{\dagger}$ & $-0.05^{\dagger}$ & $0.44^{\dagger}$ & $0.87 * * *^{\dagger}$ & & $0.78^{* * * \dagger}$ & $0.68 * * * \dagger$ \\
\hline $\mathrm{PDRP}_{\mathrm{SP}}$ & $0.46^{* *}$ & $0.41 *$ & -0.20 & $0.47 * *$ & $0.73^{* * *}$ & $0.78 * * * \dagger$ & & $0.78 * * *$ \\
\hline $\mathrm{PDRP}_{\mathrm{USA}}$ & $0.43 * *$ & $0.48^{* *}$ & -0.05 & $0.33 *$ & $0.92 * * *$ & $0.92 * * * \dagger$ & $0.78 * * *$ & \\
\hline \multicolumn{9}{|l|}{ SP data } \\
\hline & Age (HC) & Age (PD) & Disease duration & UPDRS (on) & $\mathrm{PDRP}_{\mathrm{NL}}$ & $\mathrm{PDRP}_{\mathrm{IT}}$ & $\mathrm{PDRP}_{\mathrm{SP}}$ & PDRP $_{\text {USA }}$ \\
\hline $\mathrm{PDRP}_{\mathrm{NL}}$ & 0.03 & $0.33 *$ & 0.26 & -0.01 & & $0.91 * * *$ & $0.81 * * * \dagger$ & $0.92 * * *$ \\
\hline $\mathrm{PDRP}_{\mathrm{IT}}$ & -0.02 & 0.21 & 0.25 & -0.01 & $0.91 * * *$ & & $0.77 * * * \dagger$ & $0.82 * * *$ \\
\hline $\mathrm{PDRP}_{\mathrm{SP}}$ & & $0.33^{\dagger}$ & $0.43 * \dagger$ & $0.01^{\dagger \dagger}$ & $0.81 * * * \dagger \dagger$ & $0.77 * * *{ }^{\prime} \dagger$ & & $0.84 * * * \dagger \dagger$ \\
\hline $\mathrm{PDRP}_{\text {USA }}$ & -0.11 & $0.34 *$ & 0.21 & -0.09 & $0.92 * * *$ & $0.82 * * *$ & $0.84 * * * \dagger$ & \\
\hline
\end{tabular}

* Significant at $P<0.05 ; * *$ Significant at $P<0.01 ; * * *$ Significant at $P<0.001$

$N A$ not available

${ }^{\dagger}$ Obtained from the IT validation cohort $(\mathrm{HC}=24 ; \mathrm{PD}=18)$

${ }^{\dagger}$ Obtained from the SP validation cohort $(\mathrm{PD}=30)$

related patterns (including the PDRP) with logistic regression could accurately distinguish between parkinsonian disorders. With this method, Tang et al. achieved accurate categorization of patients $(n=167)$ with an uncertain diagnosis 3-4 years before a final clinical diagnosis was made by an expert clinician masked to the imaging findings [18]. Highly similar results were obtained in an independent sample $(n=129)$ [19].

In this study, we compared data from different centers. It is well-known that variations in PET scanners and image reconstruction algorithms influence disease-related pattern scores [53-55] (supplementary Fig 1). In support of this, we recently identified clear center-specific features in the current data

Table 6 Region-weight correlations

\begin{tabular}{lllll}
\hline & PDRP $_{\mathrm{USA}}$ & $\mathrm{PDRP}_{\mathrm{IT}}$ & $\mathrm{PDRP}_{\mathrm{NL}}$ & $\mathrm{PDRP}_{\mathrm{SP}}$ \\
\hline PDRP $_{\mathrm{USA}}$ & & $0.67 * * *$ & $0.78 * * *$ & $0.481 * *$ \\
$\mathrm{PDRP}_{\mathrm{IT}}$ & $0.67 * * *$ & & $0.68 * * *$ & 0.304 \\
$\mathrm{PDRP}_{\mathrm{NL}}$ & $0.78 * * *$ & $0.68 * * *$ & & $0.458 *$ \\
$\mathrm{PDRP}_{\mathrm{SP}}$ & $0.48 * *$ & 0.30 & $0.458 *$ & \\
\hline
\end{tabular}

* Significant at $P<0.05$; * Significant at $P<0.01$; *** Significant at $P<$ 0.001 using machine-learning algorithms [56]. Therefore, PDRP subject scores cannot be compared readily between subjects from different centers. In all PDRP studies, this is solved with a $z$-transformation using the mean and standard deviation of a small healthy control group. This potentially introduces a bias, depending on which controls are selected. However, this issue is not relevant for within subject studies. Therefore, PDRP subject scores may be especially useful in tracking disease progression [14], or treatment effects [16, 35-38].

This study is methodologically different from previous PDRP studies. The PDRPs identified in this study were formed by a combination of principal components (PCs). These combinations were determined based on a forward stepwise logistic regression model [30]. There are different methods to decide which components are included in the disease-related pattern [10]. Previous studies have always identified the PDRP as PC1 in isolation [11, 20, 21]. The process of component selection is not always described in detail. Automatically choosing PC1 as the disease-related pattern, and disregarding consecutive, smaller PCs, increases the risk information loss. On the other hand, a pattern that combines multiple PCs may give a better fit of the initial sample, but may be limited in its relevance or generality across new 
Fig. 6 Subject scores for each PDRP in eight cases of MSA-p. Subject scores are $z$-transformed to NL2 controls and compared between groups with a Student's $t$ test. Bars indicate mean and standard deviation

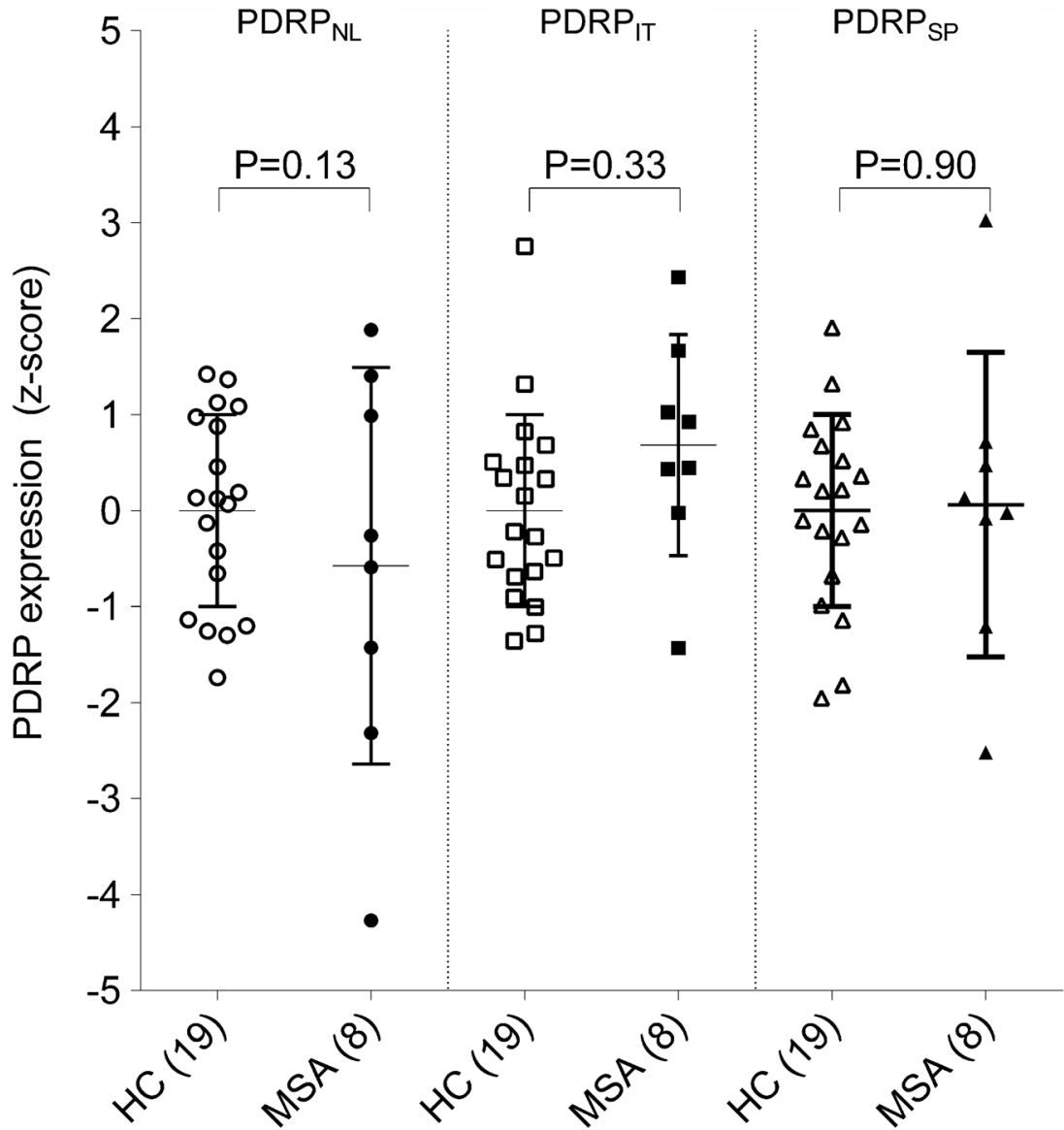

datasets. Therefore, we re-evaluated the data and included only PC1 for $\mathrm{PDRP}_{\mathrm{IT}}$, $\mathrm{PDRP}_{\mathrm{NL}}$, and $\mathrm{PDRP}_{\mathrm{SP}}$. Indeed, the $\mathrm{PC} 1$ patterns correlated better to the reference pattern $\left(\mathrm{PDRP}_{\text {USA }}\right)$. However, the patterns that included multiple PCs yielded higher diagnostic accuracy . Apart from component selection, several other decisions and cutoffs may influence pattern identification [10]. More advanced machinelearning algorithms may be of use in determining optimal patterns without the use of arbitrary thresholds and associated loss of potentially useful information [55-58].

There is increasing interest to apply the PDRP in clinical practice and in therapeutic trials [12]. However, rigorous validation by independent research groups is necessary before widespread application. The current study has contributed to the finding that the PDRP is a universal feature of $\mathrm{PD}$, and it is

Table 7 Receiver operating curve-AUCs using PC1

\begin{tabular}{lllll}
\hline & NL dataset 1 & NL dataset 2 & IT dataset & SP dataset \\
\hline HC/PD & $17 / 19$ & $19 / 20$ & $44 / 38$ & $19 / 49$ \\
PDRP $_{\text {NL-PC1 }}$ AUC & & 0.92 & 0.77 & 0.78 \\
PDRP $_{\text {IT-PC1 }}$ AUC & 0.78 & 0.95 & $0.81^{\dagger}$ & 0.72 \\
PDRP $_{\text {SP-PC1 }}$ AUC & 0.84 & 0.96 & 0.77 & \\
\hline
\end{tabular}

${ }^{\dagger}$ Obtained from the IT test cohort $(\mathrm{HC}=24 ; \mathrm{PD}=18)$ striking that such similar patterns could be identified in a limited number of ${ }^{18} \mathrm{~F}$-FDG PET scans from three populations, despite overt clinical and methodological heterogeneity. However, our results also show considerable overlap in PDRP subject scores between control and PD groups. Further study is needed to overcome this issue, perhaps by addressing potential center-specific effects in the data or by employing more advanced machine-learning algorithms.

Acknowledgements We thank Dr. David Eidelberg (Feinstein Institute for Medical Research, Manhasset, NY, USA) for providing the $\mathrm{PDRP}_{\mathrm{USA}}$ and the VOI template.

Funding information This study was funded in part by the Dutch "Stichting ParkinsonFonds." The Navarra study was supported by grants from the Government of Navarra (32/2007), Spanish Institute of Health (ISCIII) PI08/1539, and CIBERNED, Spain.

\section{Compliance with Ethical Standards}

All procedures performed in studies involving human participants were in accordance with the ethical standards of the institutional and/or national research committee and with the 1964 Helsinki declaration and its later amendments or comparable ethical standards. Ethical permission for the procedures was obtained from the local ethics committees at the University Medical Center Groningen (Groningen, The Netherlands), the University of Genoa (Genoa, Italy), and from the Ethics Committee for Medical Research of the University of Navarra (Navarra, Spain). All 
patients, or their legal representatives, and controls provided informed consent to participate in the study.

Conflict of interest The authors declare that they have no conflicts of interest.

Open Access This article is distributed under the terms of the Creative Commons Attribution 4.0 International License (http:// creativecommons.org/licenses/by/4.0/), which permits unrestricted use, distribution, and reproduction in any medium, provided you give appropriate credit to the original author(s) and the source, provide a link to the Creative Commons license, and indicate if changes were made.

\section{References}

1. Palop JJ, Chin J, Mucke L. A network dysfunction perspective on neurodegenerative diseases. Nature. 2006;443:768-73.

2. Reivich M, Kuhl D, Wolf A, Greenberg J, Phelps M, Ido T, et al. The $[18 \mathrm{~F}]$ fluorodeoxyglucose method for the measurement of local cerebral glucose utilization in man. Circ Res. 1979;44:127-37.

3. Juh R, Kim J, Moon D, Choe B, Suh T. Different metabolic patterns analysis of Parkinsonism on the 18F-FDG PET. Eur J Radiol. 2004;51:223-33.

4. Eckert T, Barnes A, Dhawan V, Frucht S, Gordon MF, Feigin AS, et al. FDG PET in the differential diagnosis of parkinsonian disorders. Neuroimage. 2005;26:912-21.

5. Teune LK, Bartels AL, de Jong BM, Willemsen AT, Eshuis SA, de Vries JJ, et al. Typical cerebral metabolic patterns in neurodegenerative brain diseases. Mov Disord. 2010;25:2395-404.

6. Huang C, Ravdin LD, Nirenberg MJ, Piboolnurak P, Severt L, Maniscalco JS, et al. Neuroimaging markers of motor and nonmotor features of Parkinson's disease: an $18 \mathrm{f}$ fluorodeoxyglucose positron emission computed tomography study. Dement Geriatr Cogn Disord. 2013;35:183-96.

7. Wang R, Xu B, Guo Z, Chen T, Zhang J, Chen Y, et al. Suite PET/ $\mathrm{CT}$ neuroimaging for the diagnosis of Parkinson's disease: statistical parametric mapping analysis. Nucl Med Commun. 2017;38: 164-9.

8. Moeller JR, Strother SC, Sidtis JJ, Rottenberg DA. Scaled subprofile model: a statistical approach to the analysis of functional patterns in positron emission tomographic data. J Cereb Blood Flow Metab. 1987;7:649-58.

9. Eidelberg D. Metabolic brain networks in neurodegenerative disorders: a functional imaging approach. Trends Neurosci. 2009;32: 548-57.

10. Spetsieris PG, Eidelberg D. Scaled subprofile modeling of resting state imaging data in Parkinson's disease: methodological issues. Neuroimage. 2011;54:2899-914.

11. Ma Y, Tang C, Spetsieris PG, Dhawan V, Eidelberg D. Abnormal metabolic network activity in Parkinson's disease: test-retest reproducibility. J Cereb Blood Flow Metab. 2007;27:597-605.

12. Schindlbeck KA, Eidelberg D. Network imaging biomarkers: insights and clinical applications in Parkinson's disease. Lancet Neurol. 2018;17:629-40.

13. Holtbernd F, Ma Y, Peng S, Schwartz F, Timmermann L, Kracht L, et al. Dopaminergic correlates of metabolic network activity in Parkinson's disease. Hum Brain Mapp. 2015;36:3575-85.

14. Huang C, Tang C, Feigin A, Lesser M, Ma Y, Pourfar M, et al. Changes in network activity with the progression of Parkinson's disease. Brain. 2007;130:1834-46.

15. Niethammer M, Eidelberg D. Metabolic brain networks in translational neurology: concepts and Applications. Ann Neurol. 2012;72(5):635-47.
16. Asanuma K, Tang C, Ma Y, Dhawan V, Mattis P, Edwards C, et al. Network modulation in the treatment of Parkinson's disease. Brain. 2006;129:2667-78.

17. Holtbernd F, Gagnon JF, Postuma RB, Ma Y, Tang CC, Feigin A, et al. Abnormal metabolic network activity in REM sleep behavior disorder. Neurology. 2014;82:620-7.

18. Tang CC, Poston KL, Eckert T, Feigin A, Frucht S, Gudesblatt M, et al. Differential diagnosis of parkinsonism: a metabolic imaging study using pattern analysis. Lancet Neurol. 2010;9:149-58.

19. Tripathi M, Tang CC, Feigin A, De Lucia I, Nazem A, Dhawan V, et al. Automated differential diagnosis of early parkinsonism using metabolic brain networks: a validation study. J Nucl Med. 2016;57(1):60-6.

20. Wu P, Wang J, Peng S, Ma Y, Zhang H, Guan Y, et al. Metabolic brain network in the Chinese patients with Parkinson's disease based on 18F-FDG PET imaging. Parkinsonism Relat Disord. 2013;19:622-7.

21. Tomse P, Jensterle L, Grmek M, Zaletel K, Pirtosek Z, Dhawan V, et al. Abnormal metabolic brain network associated with Parkinson's disease: replication on a new European sample. Neuroradiology. 2017;59:507-15.

22. Matthews DC, Lerman H, Lukic A, Andrews RD, Mirelman A, Wernick MN, et al. FDG PET Parkinson's disease-related pattern as a biomarker for clinical trials in early stage disease. Neuroimage Clin. 2018;20:572-9.

23. Teune LK, Renken RJ, Mudali D, De Jong BM, Dierckx RA, Roerdink JB, et al. Validation of parkinsonian disease-related metabolic brain patterns. Mov Disord. 2013;28:547-51.

24. Teune LK, Renken RJ, de Jong BM, Willemsen AT, van Osch MJ, Roerdink JB, et al. Parkinson's disease-related perfusion and glucose metabolic brain patterns identified with PCASL-MRI and FDG-PET imaging. Neuroimage Clin. 2014;5:240-4.

25. Meles SK, Vadasz D, Renken RJ, Sittig-Wiegand E, Mayer G, Depboylu C, et al. FDG PET, dopamine transporter SPECT, and olfaction: combining biomarkers in REM sleep behavior disorder. Mov Disord. 2017;32:1482-6.

26. Postuma RB, Berg D, Stern M, Poewe W, Olanow CW, Oertel W, et al. MDS clinical diagnostic criteria for Parkinson's disease. Mov Disord. 2015;30:1591-601.

27. Arnaldi D, Morbelli S, Brugnolo A, Girtler N, Picco A, Ferrara M, et al. Functional neuroimaging and clinical features of drug naive patients with de novo Parkinson's disease and probable RBD. Parkinsonism Relat Disord. 2016;29:47-53.

28. Garcia-Garcia D, Clavero P, Gasca Salas C, Lamet I, Arbizu J, Gonzalez-Redondo R, et al. Posterior parietooccipital hypometabolism may differentiate mild cognitive impairment from dementia in Parkinson's disease. Eur J Nucl Med Mol Imaging. 2012;39:1767-77.

29. Della Rosa PA, Cerami C, Gallivanone F, Prestia A, Caroli A, Castiglioni I, et al. A standardized [18F]-FDG-PET template for spatial normalization in statistical parametric mapping of dementia. Neuroinformatics. 2014;12:575-93.

30. Akaike H. A new look at the statistical model identification. IEEE Trans Autom Control. 1974;19(6):716-23.

31. Habeck C, Foster NL, Perneczky R, Kurz A, Alexopoulos P, Koeppe RA, et al. Multivariate and univariate neuroimaging biomarkers of Alzheimer's disease. Neuroimage. 2008;40:1503-15.

32. Eidelberg D, Moeller JR, Dhawan V, Spetsieris P, Takikawa S, Ishikawa $\mathrm{T}$, et al. The metabolic topography of parkinsonism. J Cereb Blood Flow Metab. 1994;14:783-801.

33. Rodriguez-Oroz MC, Jahanshahi M, Krack P, Litvan I, Macias R, Bezard E, et al. Initial clinical manifestations of Parkinson's disease: features and pathophysiological mechanisms. Lancet Neurol. 2009;8:1128-39.

34. DeLong MR, Wichmann T. Circuits and circuit disorders of the basal ganglia. Arch Neurol. 2007;64:20-4. 
35. Su PC, Ma Y, Fukuda M, Mentis MJ, Tseng HM, Yen RF, et al. Metabolic changes following subthalamotomy for advanced Parkinson's disease. Ann Neurol. 2001;50:514-20.

36. Trost M, Su S, Su P, Yen RF, Tseng HM, Barnes A, et al. Network modulation by the subthalamic nucleus in the treatment of Parkinson's disease. Neuroimage. 2006;31:301-7.

37. Wang J, Ma Y, Huang Z, Sun B, Guan Y, Zuo C. Modulation of metabolic brain function by bilateral subthalamic nucleus stimulation in the treatment of Parkinson's disease. J Neurol. 2010;257:728.

38. Lin TP, Carbon M, Tang C, Mogilner AY, Sterio D, Beric A, et al. Metabolic correlates of subthalamic nucleus activity in Parkinson's disease. Brain. 2008;131:1373-80.

39. Berti V, Polito C, Ramat S, Vanzi E, De Cristofaro MT, Pellicano G, et al. Brain metabolic correlates of dopaminergic degeneration in de novo idiopathic Parkinson's disease. Eur J Nucl Med Mol Imaging. 2010;37:537-44.

40. Ko JH, Lerner RP, Eidelberg D. Effects of levodopa on regional cerebral metabolism and blood flow. Mov Disord. 2015;30:54-63.

41. Gasca-Salas C, Clavero P, Garcia-Garcia D, Obeso JA, RodriguezOroz MC. Significance of visual hallucinations and cerebral hypometabolism in the risk of dementia in Parkinson's disease patients with mild cognitive impairment. Hum Brain Mapp. 2016;37: 968-77.

42. Hellwig S, Amtage F, Kreft A, Buchert R, Winz OH, Vach W, Spehl TS, Rijntjes M, Hellwig B, Weiller C, Winkler C, Weber WA, Tuscher O, Meyer PT. (1)(8)F]FDG-PET is superior to $[(1)(2)(3) I] I B Z M-S P E C T$ for the differential diagnosis of parkinsonism. Neurology 2012;79:1314-22.

43. Tripathi M, Dhawan V, Peng S, Kushwaha S, Batla A, Jaimini A, et al. Differential diagnosis of parkinsonian syndromes using F-18 fluorodeoxyglucose positron emission tomography. Neuroradiology. 2013;55:483-92.

44. Brajkovic L, Kostic V, Sobic-Saranovic D, Stefanova E, JecmenicaLukic M, Jesic A, et al. The utility of FDG-PET in the differential diagnosis of parkinsonism. Neurol Res. 2017;39:675-84.

45. Meyer PT, Frings L, Rucker G, Hellwig S. (18)F-FDG PET in parkinsonism: differential diagnosis and evaluation of cognitive impairment. J Nucl Med. 2017;58:1888-98.

46. Meles SK, Teune LK, de Jong BM, Dierckx RA, Leenders KL. Metabolic imaging in parkinson disease. J Nucl Med. 2017;58: 23-8.

47. Ishibashi K, Onishi A, Fujiwara Y, Oda K, Ishiwata K, Ishii K. Longitudinal effects of aging on (18)F-FDG distribution in cognitively normal elderly individuals. Sci Rep. 2018;8(1):11557.

48. Zhang N, Gordon ML, Ma Y, Chi B, Gomar JJ, Peng S, et al. The age-related perfusion pattern measured with arterial spin labeling MRI in healthy subjects. Front Aging Neurosci. 2018;10:214.
49. Moeller JR, Ishikawa T, Dhawan V, Spetsieris P, Mandel F, Alexander GE, et al. The metabolic topography of normal aging. J Cereb Blood Flow Metab. 1996;16:385-98.

50. Moeller JR, Eidelberg D. Divergent expression of regional metabolic topographies in Parkinson's disease and normal ageing. Brain. 1997;120(Pt 12):2197-206.

51. Rizzo G, Copetti M, Arcuti S, Martino D, Fontana A, Logroscino G. Accuracy of clinical diagnosis of Parkinson disease: a systematic review and meta-analysis. Neurology. 2016;86:566-76.

52. Ko JH, Lee CS, Eidelberg D. Metabolic network expression in parkinsonism: clinical and dopaminergic correlations. J Cereb Blood Flow Metab. 2017;37(2):683-693.

53. Tomse P, Jensterle L, Rep S, Grmek M, Zaletel K, Eidelberg D, et al. The effect of 18F-FDG-PET image reconstruction algorithms on the expression of characteristic metabolic brain network in Parkinson's disease. Phys Med. 2017;41:129-35.

54. Tomse P, Peng S, Pirtosek Z, Zaletel K, Dhawan V, Eidelberg D, et al. The effects of image reconstruction algorithms on topographic characteristics, diagnostic performance and clinical correlation of metabolic brain networks in Parkinson's disease. Phys Med. 2018;52:104-12.

55. Kogan RV, de Jong BA, Renken RJ, Meles SK, van Snick PJH, Golla S, et al. Factors affecting the harmonization of disease-related metabolic brain pattern expression quantification in [(18)F]FDGPET (PETMETPAT). Alzheimers Dement (Amst). 2019;11:47282.

56. van Veen R, Talavera Martinez L, Kogan RV, Meles SK, Mudali D, Roerdink JBTM, Massa F, Grazzini M, Obeso JA, Rodriguez-Oroz MC, Leenders KL, Renken RJ, de Vries JJG, Biehl M. Machine learning based analysis of FDG-PET image data for the diagnosis of neurodegenerative diseases. Application of Intelligent Systems (APPIS) 2018;in press.

57. Mudali D, Teune LK, Renken RJ, Leenders KL, Roerdink JB. Classification of parkinsonian syndromes from FDG-PET brain data using decision trees with SSM/PCA features. Comput Math Methods Med. 2015;2015:136921.

58. Mudali D, Biehl M, Meles SK, Renken RJ, Garcia-Garcia D, Clavero P, et al. Differentiating early and late stage Parkinson's disease from healthy controls. JBEMi. 2016;3(6):33-43.

59. Manzanera OM, Meles SK, Leenders KL, Renken RJ, Pagani M, Arnaldi D, Nobili F, Obeso J, Oroz MR, Morbelli S, Maurits NM. Scaled Subprofile Modeling and Convolutional Neural Networks for the Identification of Parkinson's Disease in 3D Nuclear Imaging Data. International Journal of Neural Systems. (2019);29(9).

Publisher's note Springer Nature remains neutral with regard to jurisdictional claims in published maps and institutional affiliations. 